\title{
Genomic analysis and plant growth-promoting potential of a Serratia marcescens
}

\section{isolated from food}

\author{
Análise genômica e potencial de promoção de crescimento vegetal de uma Serratia marcescens \\ isolada de alimento
}

Análisis genómico y potencial de promoción del crecimiento vegetal de una Serratia marcescens

aislada de alimento

Received: 12/15/2021 | Reviewed: 12/22/2021 | Accept: 12/29/2021| Published: 01/07/2022

\author{
Cristiane Rodrigues Silva \\ ORCID: https://orcid.org/0000-0002-6306-4998 \\ Universidade Federal do Estado do Rio de Janeiro, Brazil \\ E-mail: cristiane.silva@unirio.br \\ Rafael Monção Miller \\ ORCID: https://orcid.org/0000-0003-2760-8748 \\ Universidade Federal do Estado do Rio de Janeiro, Brazil \\ E-mail: moncaomiller@gmail.com \\ Bárbara Costa Peixoto \\ ORCID: https://orcid.org/0000-0002-8228-0194 \\ Universidade Federal do Estado do Rio de Janeiro, Brazil \\ E-mail: baabicp@gmail.com \\ Lílian Aveleda \\ ORCID: https://orcid.org/0000-0002-0876-0974 \\ Universidade Federal do Estado do Rio de Janeiro, Brazil \\ E-mail: lili.aveleda@gmail.com \\ Victor Augustus Marin \\ ORCID: https://orcid.org/0000-0002-9827-6552 \\ Universidade Federal do Estado do Rio de Janeiro, Brazil \\ E-mail: victor.marin@unirio.br
}

\begin{abstract}
A genomic analysis of the potential application of a Serratia marcescens strain in the plant-growth promotion. We performed whole-genome sequencing of Serratia marcescens isolated from a Minas Frescal Cheese. The genomic repertoire revealed a bacterium of agricultural and biotechnological interest. In the plant-growth promotion traits, we highlight genes encoding proteins possibly responsible for the biosynthesis of phytohormone indole acetic acid, organic compounds that act in iron uptake, and the Phosphate solubilization system. Genes encoding for enzymes like the versatile L-asparaginase stimulates the development of seeds and grains and can benefit the food industry due to a mitigation effect on acrylamide and notably, has medical applications as a chemotherapeutic agent or is applicable by its antimicrobial and anti-inflammatory properties. Moreover, functional diversity of genes encoding for resistance to different metals and metabolism of xenobiotics genes can be found in this strain, reinforcing its biotechnological potential. The versatile enzymes that can be produced by $S$. marcescens benefit the food, pharmaceutical, textile, agronomic, and cosmetic industries. The relevant genetic systems of $S$. marcescens described here may be used to promote plant growth and health and improve the environment. To the best of our knowledge, this is the first genome sequence report on $S$. marcescens isolated from cheese, with potential application as promoting plant growth and providing a baseline for future genomic studies on the development of this species.
\end{abstract}

Keywords: Serratia marcescens; Plant growth-promotion; Biotechnology.

\section{Resumo}

Análise genômica do potencial de aplicação de uma cepa de Serratia marcescens na promoção de crescimento vegetal. Foi realizado o sequenciamento do genoma completo de Serratia marcescens isolada de queijo Minas Frescal. O repertório genômico revelou uma bactéria de interesse agrícola e biotecnológico. Dentre as características de promoção do crescimento de plantas, destacamos genes que codificam proteínas possivelmente responsáveis pela biossíntese do fitohormônio ácido indol acético, compostos orgânicos que atuam na captação de ferro e o sistema de solubilização de Fosfato. Genes que codificam enzimas como a versátil L-asparaginase estimulam o desenvolvimento de sementes e grãos e podem beneficiar a indústria de alimentos devido ao efeito de mitigação da acrilamida e, notadamente, tem aplicações médicas como agente quimioterápico ou é aplicável por seus agentes antimicrobianos e propriedades anti-inflamatórias. Além disso, uma diversidade funcional de genes que codificam para resistência a 
diferentes metais e metabolismo de xenobióticos pôde ser encontrada nesta linhagem, reforçando seu potencial biotecnológico. As enzimas versáteis que podem ser produzidas em $S$. marcescens beneficiam as indústrias alimentícia, farmacêutica, têxtil, agronômica e cosmética. Os sistemas genéticos relevantes de S. marcescens descritos aqui podem ser usados para promover o crescimento e a saúde das plantas e melhorar o meio ambiente. Até onde sabemos, este é o primeiro relato de sequência de genoma de $S$. marcescens isolado de queijo, com potencial aplicação como promotor de crescimento de plantas e fornecendo uma linha de base para futuros estudos genômicos sobre o desenvolvimento desta espécie.

Palavras-chave: Serratia marcescens; Promoção de crescimento vegetal; Biotecnologia.

\section{Resumen}

Un análisis genómico de la posible aplicación de una cepa de Serratia marcescens en la promoción del crecimiento vegetal. Métodos: Se realizó la secuenciación del genoma completo de Serratia marcescens aislada de un queso Minas Frescal. El repertorio genómico revelo una bacteria de interés agrícola y biotecnológico. En los rasgos de promoción del crecimiento de las plantas, destacamos los genes que codifican proteínas posiblemente responsables de la biosíntesis del ácido acético indol fitohormono, compuestos orgánicos que actúan en la absorción de hierro y el sistema de solubilización de fosfato. Los genes que codifican para enzimas como la versátil L-asparaginasa estimulan el desarrollo de semillas y granos y pueden beneficiar a la industria alimentaria debido a un efecto de mitigación sobre la acrilamida y, en particular, tiene aplicaciones médicas como agente quimioterapéutico o es aplicable por sus propiedades antimicrobianas y antiinflamatorias. Además, una diversidad funcional de genes que codifican para la resistencia a diferentes metales y el metabolismo de los genes xenobióticos se puede encontrar en esta cepa, reforzando su potencial biotecnológico. Las enzimas versátiles que pueden ser producidas por Serratia marcescens benefician a las industrias alimentaria, farmacéutica, textil, agronómica y cosmética. Los sistemas genéticos relevantes de $S$. marcescens descritos aquí pueden utilizarse para promover el crecimiento y la salud de las plantas y mejorar el medio ambiente. Hasta donde sabemos, este es el primer informe de secuencia del genoma de $S$. marcescens aislado del queso, con potencial aplicación como promotor del crecimiento vegetal y proporcionando una línea de base para futuros estudios genómicos sobre el desarrollo de esta especie.

Palabras clave: Serratia marcescens; Promoción del crecimiento vegetal; Biotecnología.

\section{Introduction}

Serratia marcescens, classified under the family Yersiniaceae of the order Enterobacterales (Adeolu et al., 2016), is capable of thriving in a broad range of environments.

The wide variety of gene repertoire enables $S$. marcescens to be a ubiquitous microorganism, successful in diverse environments and with multipurpose applications or effects. For example, marine $S$. marcescens demonstrated antioxidant and antibacterial activity against some Gram-positive and Gram-negative bacteria (Gangadharan et al., 2020). Moreover, oil spills in aquatic ecosystems can be bioremediated by a biodispersant produced by $S$. marcescens characterized by low toxicity, high biodegradability, and good ecological acceptability (dos Santos et al., 2021).

In the terrestrial environment, the species can act as a pathogen to animals (Friman et al., 2019; Ishii et al., 2012). However is agronomically relevant with its antifungal effect (Troskie et al., 2014). Furthermore, it can be considered a biocontrol agent with herbicidal activity (Kamran et al., 2017) and as a plant growth-promoting rhizobacteria (PGPR), improving the health and development of their host plant by acting on the solubilization of inorganic $\mathrm{P}$ present in the soil (Rodríguez \& Fraga, 1999).

Alternative approaches can improve the soil and global water healthy, once continuously contaminated by the human being, to maintain the exorbitant life cycle. Due use of bacteria systems in biotechnology plus the promise $o f$. marcescens role in several mechanisms, it could be used in different scenarios, including azo dyes degradation to soil decontamination (Mahmood et al., 2017).

Functional diversity of genes encoding for enzymes have evidenced an important assignment for S. marcescens in the biotechnological industry, including segments such as food, cosmetics, chemical, and pharmaceutical (dos Santos et al., 2021; Falade \& Ekundayo, 2021). 
Both disease and therapy are present in the dual role of S. marcescens in the clinic, responsible for outbreaks (Cristina et al., 2019) was even identified as an opportunist in outbreaks during covid-19 treatment (Amarsy et al., 2020). However, it has shown an extensive presence of enzymes with antimicrobial and anti-inflammatory properties proving effective to even resistant microorganisms and with the possibility of use as a chemotherapeutic agent in different cancer cell lines (AbdelRazik et al., 2019; Pavithrra \& Rajasekaran, 2020).

The broad niche and functional diversity of the $S$. marcescens are probably influenced by and, at the same time, influence on the highly dynamic genome (Cristina et al., 2019), turning relevant the periodical investigation of the genetic background of emerging $S$. marcescens strains from various sources. Moreover, the potential application of the strain to promote plant growth was explored from the genotypic perspective.

\section{Material and Methods}

\subsection{Isolation and characterization of the Serratia marcescens}

The Serratia marcescens strain was isolated from a Gram-negative pool of bacteria from a previous analysis of Minas Frescal Cheese according to the methodology of Silva et al., 2020. Subsequently, the strain was isolated in Mueller Hinton Agar. Genomic DNA of the bacterial strain was extracted with a NucleoSpin Tissue kit (Macherey-Nagel \& Germany, 2018) in conformity with the manufacturer's protocol.

\subsection{Whole genome sequence analysis}

A genomic library was constructed using a Nextera XT Kit (Illumina Inc., San Diego, C) by Genone Company. Total genomic DNA was sequenced using a MiSeq platform from Illumina Inc. (Genone Company). The sequence was assembled and the draft genome using Prokka (Seemann, 2014) in the Galaxy site https://www.usegalaxy.org and RAST (Brettin et al., 2015) in the site http://rast.nmpdr.org/rast.cgi. The DFAST - Prokaryotic genome annotation pipeline from the site https://dfast.ddbj.nig.ac.jp/dfc/ (Tanizawa et al., 2016; Tanizawa et al., 2018) and PATRIC in the site https://www.patricbrc.org/ (Davis et al., 2020).

\section{Results}

\subsection{Characterization of the Serratia marcescens}

The bacterial strain was identified to be Serratia marcescens and was deposited at the Genbank under the number SUB9616311, BioProject and Biosample accession number are PRJNA729465 and SAMN19116778, respectively. The genome has Total Length (bp) 4,969,854 and GC Content 59.6\%; 4,722 CD; 4 rRNA; 81 tRNA; 2 CRISPR.

\subsection{Genomic repertoire}

The diversified genomic repertoire of $S$. marcescens made it possible to group them into different sets of genes according to the benefits presented. Table 1 presents genes related to several systems involved in plant-growth promotion. 
Table 1 - Genes involved in the plant growth promotion traits.

\begin{tabular}{|c|c|c|c|}
\hline Location & Gene & Product & Pathway \\
\hline $71101 . .72780$ & ipdC1 & Indole-3-pyruvate decarboxylase & \multirow{6}{*}{$\begin{array}{c}\text { Synthesis of the } \\
\text { phytohormone indole } \\
\text { acetic acid (IAA) }\end{array}$} \\
\hline 1745616..1747277 & ipdC2 & Indole-3-pyruvate decarboxylase & \\
\hline 1348409..1349596 & tyrB1 & Aromatic-amino-acid aminotransferase & \\
\hline 2400258..2401487 & tyrB2 & Aromatic-amino-acid aminotransferase & \\
\hline $841137 . .842327$ & $\operatorname{aspC}$ & aspartate aminotransferase & \\
\hline 1317606..1319021 & - & Aromatic-L-amino-acid decarboxylase & \\
\hline $3385706 . .3386512$ & $\operatorname{trpA}$ & tryptophan synthase alpha chain & \multirow{8}{*}{$\begin{array}{l}\text { Tryptophan biosynthesis } \\
\text { pathway }\end{array}$} \\
\hline 3386512..3387702 & $\operatorname{trpB}$ & tryptophan synthase beta chain & \\
\hline 33877443380105 & $\operatorname{trn} C$ & bifunctional indole-3-glycerol phosphate & \\
\hline $500 / / 44 . .5309105$ & trpe & synthase/phosphoribosylanthranilate isomerise & \\
\hline 3389109..3390107 & $\operatorname{trpD}$ & anthranilate phosphoribosyltransferase & \\
\hline 3390124..3390705 & trpG & anthranilate synthase component 2 & \\
\hline 3390705..3392267 & $\operatorname{trpE}$ & anthranilate synthase component 1 & \\
\hline $3392647 . .3393528$ & $\operatorname{trpH}$ & hypothetical protein & \\
\hline 1346991..1348388 & $g a b P$ & GABA permease & GABA transport \\
\hline $2555735 . .2557189$ & $g a b D$ & $\begin{array}{l}\text { Succinate-semialdehyde dehydrogenase [NADP(+)] } \\
\text { GabD }\end{array}$ & GABA degradation \\
\hline $1496042 . .1497370$ & puuA1 & Gamma-glutamylputrescine synthetase PuuA & \multirow{13}{*}{ GABA biosynthesis } \\
\hline 2065828..2067198 & puuA2 & Gamma-glutamylputrescine synthetase PuuA & \\
\hline $3048881 . .3050299$ & puuA3 & Gamma-glutamylputrescine synthetase PuuA & \\
\hline 2035575..2036876 & puuB1 & Gamma-glutamylputrescine oxidoreductase & \\
\hline $3044542 . .3045822$ & puuB2 & Gamma-glutamylputrescine oxidoreductase & \\
\hline 3045834..3047330 & puuC & NADP/NAD-dependent aldehyde dehydrogenase PuuC & \\
\hline $3047900 . .3048661$ & puuD & Gamma-glutamyl-gamma-aminobutyrate hydrolase PuuD & \\
\hline $2554457 . .2555722$ & puuE & 4-aminobutyrate aminotransferase PuuE & \\
\hline 1497856..1498413 & puuR1 & HTH-type transcriptional regulator PuuR & \\
\hline $3047346 . .3047903$ & puuR2 & HTH-type transcriptional regulator PuuR & \\
\hline $4129206 . .4130669$ & prr1 & Gamma-aminobutyraldehyde dehydrogenase & \\
\hline 4134757..4136181 & prr2 & Gamma-aminobutyraldehyde dehydrogenase & \\
\hline $707261 . .708193$ & gbuA & Guanidinobutyrase & \\
\hline 751743..752852 & potF & Putrescine-binding periplasmic protein & \multirow{7}{*}{ Polyamine transport } \\
\hline $2139577 . .2140893$ & potE & Putrescine transporter PotE & \\
\hline 2998784..2999827 & $\operatorname{pot} D$ & $\begin{array}{l}\text { Spermidine/putrescine transport system substrate-binding } \\
\text { protein PotD }\end{array}$ & \\
\hline $3003984 . .3004766$ & potC & $\begin{array}{l}\text { Spermidine/putrescine transport system permease protein } \\
\text { PotC }\end{array}$ & \\
\hline $3004763 . .3005623$ & pot $B$ & $\begin{array}{l}\text { Spermidine/putrescine transport system permease protein } \\
\text { PotB }\end{array}$ & \\
\hline $3005607 . .3006722$ & potA & $\begin{array}{l}\text { Spermidine/putrescine transport system ATP-binding } \\
\text { protein PotA }\end{array}$ & \\
\hline $3050608 . .3051978$ & puиP & Putrescine importer & \\
\hline $2229642 . .2230538$ & miaA & tRNA dimethylallyltransferase & \multirow[b]{2}{*}{ Cytokinin biosynthesis } \\
\hline 281151..282575 & $\operatorname{mia} B$ & $\begin{array}{l}\text { tRNA-2-methylthio-N(6)-dimethylallyladenosine } \\
\text { synthase }\end{array}$ & \\
\hline $2812914 . .2814890$ & speA & Biosynthetic arginine decarboxylase & \multirow{8}{*}{ Putrescine biosynthesis } \\
\hline $2815085 . .2816005$ & speB & Agmatinase & \\
\hline 2732182..2734347 & speC & Ornithine decarboxylase & \\
\hline 2772843..2773637 & speD & S-adenosylmethionine decarboxylase proenzyme & \\
\hline $2773665 . .2774528$ & speE1 & Polyamine aminopropyltransferase & \\
\hline $3724779 . .3725567$ & speE2 & Polyamine aminopropyltransferase & \\
\hline 2137352..2139514 & speF & Inducible ornithine decarboxylase & \\
\hline $2810851 . .2812005$ & metK & S-adenosylmethionine synthase & \\
\hline $4488192 . .4490330$ & $\operatorname{cadA}$ & Inducible lysine decarboxylase & \multirow{3}{*}{$\begin{array}{l}\text { Cadaverine, putrescine } \\
\text { biosynthesis }\end{array}$} \\
\hline 4486753..4488096 & cadB & putative cadaverine/lysine antiporter & \\
\hline $4484830 . .4486383$ & cadC & Transcriptional activator CadC & \\
\hline
\end{tabular}




\begin{tabular}{|c|c|c|c|}
\hline 2772843..2773637 & speD & S-adenosylmethionine decarboxylase proenzyme & \multirow{6}{*}{$\begin{array}{l}\text { Spermidine/Spermine } \\
\text { biosynthesis }\end{array}$} \\
\hline $2773665 . .2774528$ & speE1 & Polyamine aminopropyltransferase & \\
\hline 3724779..3725567 & speE2 & Polyamine aminopropyltransferase & \\
\hline $906353 . .906919$ & speG1 & Spermidine N(1)-acetyltransferase & \\
\hline 1734415..1734954 & speG2 & Spermidine N(1)-acetyltransferase & \\
\hline $4243961 . .4244512$ & speG3 & Spermidine N(1)-acetyltransferase & \\
\hline $3336851 . .3338128$ & chiAl & Chitinase & \multirow{4}{*}{ Chitin degradation } \\
\hline $3889421 . .3891112$ & chiA & Chitinase & \\
\hline 4797788..4799287 & $\operatorname{chiB}$ & Chitinase & \\
\hline $286807 . .288246$ & Chid & Chitinase & \\
\hline $4841474 . .4842253$ & budA & Alpha-acetolactate decarboxylase & \multirow{9}{*}{ Acetoin Synthesis } \\
\hline $4842286 . .4843971$ & budB & Acetolactate synthase, catabolic & \\
\hline 1902321..1904015 & ilvB1 & Acetolactate synthase isozyme 1 large subunit & \\
\hline $2829574 . .2831214$ & ilvB2 & Acetolactate synthase isozyme 1 large subunit & \\
\hline 4878208..4879854 & $i l v G$ & Acetolactate synthase isozyme 2 large subunit & \\
\hline 1882791..1884509 & ilvI & Acetolactate synthase isozyme 3 large subunit & \\
\hline 1904019..1904312 & ilvN & Acetolactate synthase isozyme 1 small subunit & \\
\hline 4877954..4878211 & ilvM & Acetolactate synthase isozyme 2 small subunit & \\
\hline $1882297 . .1882788$ & ilvH & Acetolactate synthase isozyme 3 small subunit & \\
\hline 1395126..1396316 & entCl & Isochorismate synthase & \multirow{11}{*}{ Siderophore Production } \\
\hline $2301840 . .2303045$ & entC2 & Isochorismate synthase & \\
\hline $2300204 . .2301832$ & entE & Enterobactin synthase subunit E & \\
\hline $2304690 . .2308634$ & entF & Enterobactin synthase subunit $\mathrm{F}$ & \\
\hline $2299340 . .2300200$ & entB & Enterobactin synthase subunit B & \\
\hline $2303257 . .2304513$ & entS1 & Enterobactin exporter & \\
\hline $3630309 . .3631592$ & entS2 & Enterobactin exporter & \\
\hline $2050892 . .2051299$ & exbD1 & Biolpolymer transporter protein & \\
\hline 2623298..2623714 & exbD2 & Biolpolymer transporter protein & \\
\hline $2622308 . .2623288$ & $\operatorname{exbB}$ & Biolpolymer transporter protein & \\
\hline $4916366 . .4916845$ & $B f r$ & Bacterioferritin & \\
\hline $2540910 . .2542397$ & uxaA & Altronate dehydrolase & \multirow{3}{*}{$\begin{array}{l}\text { Plant polymer } \\
\text { degradation enzymes }\end{array}$} \\
\hline $2539477 . .2540889$ & uxaC & Uronate isomerise & \\
\hline $3907226 . .3908329$ & $b c s Z$ & Endoglucanase (cellulase) & \\
\hline 1361417..1363789 & Gcd & Quinoprotein glucose dehydrogenase & \multirow{3}{*}{ Inorganic $P$ solubilisation } \\
\hline $2197430 . .2197960$ & $P p a$ & Inorganic pyrophosphatase & \\
\hline 4233863..4235413 & $P p x$ & Exopolyphosphatase & \\
\hline 1113239..1114927 & appA1 & Oligopeptide-binding protein AppA & \multirow{14}{*}{$\begin{array}{c}\text { Organic P mineralization } \\
\text { and glyphosate } \\
\text { degradation }\end{array}$} \\
\hline 1534333..1536141 & appA2 & Oligopeptide-binding protein AppA & \\
\hline $2127176 . .2127904$ & phnF & phosphonate metabolism transcriptional regulator $\mathrm{PhnF}$ & \\
\hline $2127905 . .2128348$ & phnG & phosphonate C-P lyase system protein PhnG & \\
\hline $2128352 . .2128933$ & phnH & $\begin{array}{l}\text { Alpha-D-ribose 1-methylphosphonate 5-triphosphate } \\
\text { synthase subunit } \mathrm{PhnH}\end{array}$ & \\
\hline 2128933..2130018 & phnI & $\begin{array}{l}\text { Alpha-D-ribose 1-methylphosphonate 5-triphosphate } \\
\text { synthase subunit PhnI }\end{array}$ & \\
\hline $2130011 . .2130871$ & phnJ & $\begin{array}{l}\text { Alpha-D-ribose 1-methylphosphonate 5-phosphate C-P } \\
\text { lyase }\end{array}$ & \\
\hline 2130871..2131659 & $\operatorname{phnK}$ & $\begin{array}{l}\text { Putative phosphonates utilization ATP-binding protein } \\
\text { PhnK }\end{array}$ & \\
\hline $2131676 . .2132380$ & phnL & $\begin{array}{l}\text { Alpha-D-ribose 1-methylphosphonate } \\
\text { 5-triphosphate synthase subunit } \mathrm{PhnL}\end{array}$ & \\
\hline $2132380 . .2133516$ & phnM & $\begin{array}{l}\text { Alpha-D-ribose 1-methylphosphonate 5-triphosphate } \\
\text { diphosphatase }\end{array}$ & \\
\hline $2133516 . .2134085$ & $p h n N$ & Ribose 1,5-bisphosphate phosphokinase PhnN & \\
\hline $254558 . .254986$ & phnO & Aminoalkylphosphonate $\mathrm{N}$-acetyltransferase & \\
\hline $2134697 . .2135479$ & phnP & Phosphoribosyl 1,2-cyclic phosphate phosphodiesterase & \\
\hline $3102067 . .3103170$ & phnW & 2-aminoethylphosphonate--pyruvate transaminase & \\
\hline
\end{tabular}




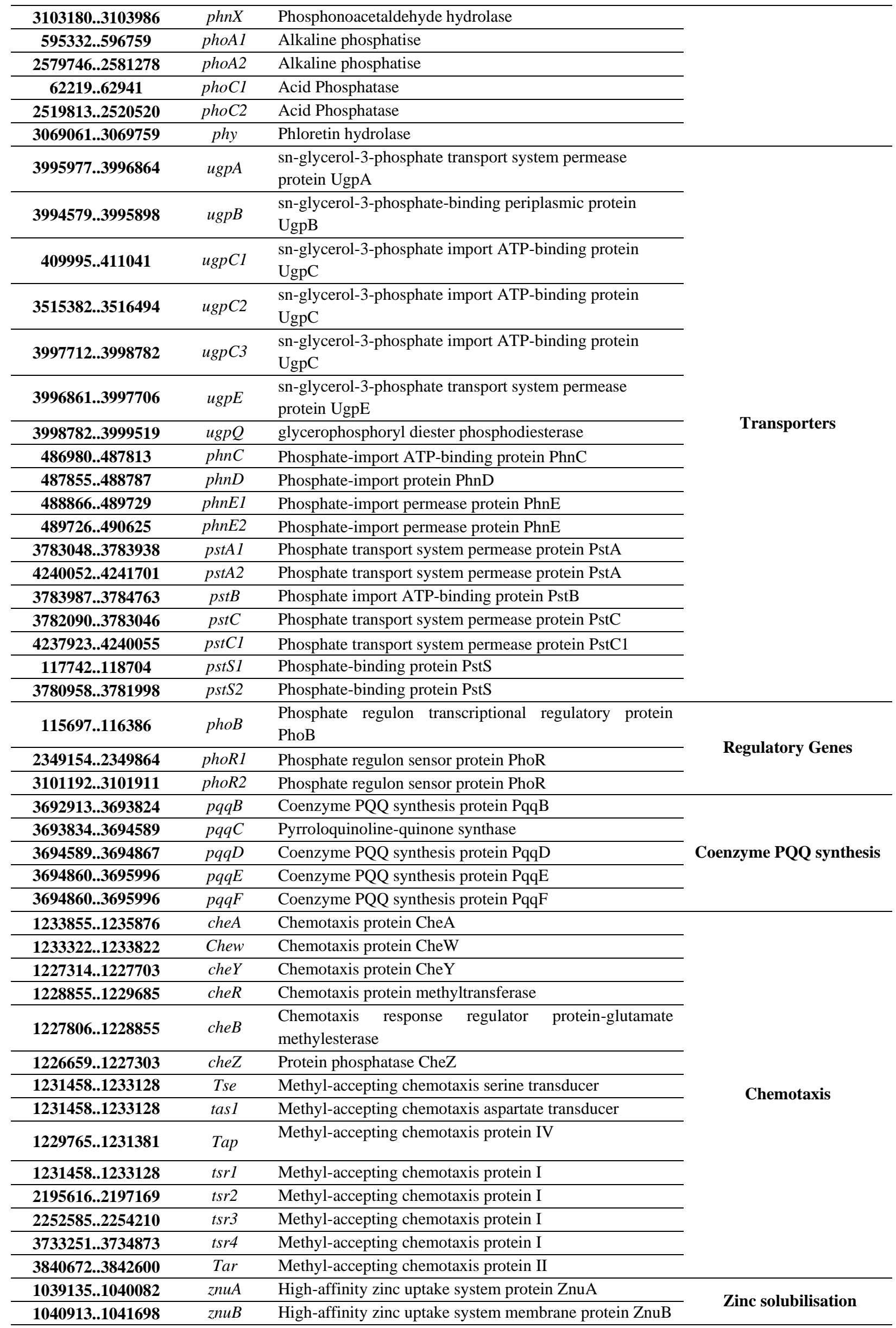




\begin{tabular}{|c|c|c|c|}
\hline 183244..183957 & $z n u C 1$ & $\begin{array}{l}\text { High-affinity zinc uptake system ATP-binding protein } \\
\text { ZnuC }\end{array}$ & \\
\hline 1040158..1040916 & $z n u C 2$ & $\begin{array}{l}\text { High-affinity zinc uptake system ATP-binding protein } \\
\text { ZnuC }\end{array}$ & \\
\hline 110912..3111802 & $z n u C 3$ & $\begin{array}{l}\text { High-affinity zinc uptake system ATP-binding protein } \\
\text { ZnuC }\end{array}$ & \\
\hline 3532752..3534362 & pitAl & Low-affinity inorganic phosphate transporter 1 & \\
\hline $4649865 . .4651367$ & pitA2 & Low-affinity inorganic phosphate transporter 1 & \\
\hline 4488192..4490330 & $\operatorname{cadA}$ & Inducible lysine decarboxylase & \\
\hline 4486753..4488096 & $\operatorname{cadB}$ & putative cadaverine/lysine antiporter & \\
\hline $4484830 . .4486383$ & cadC & Transcriptional activator CadC & \\
\hline 3972332..3974650 & $z n t A$ & Zinc/cadmium/lead-transporting P-type ATPase & \\
\hline $3469580 . .3470563$ & $z n t B 1$ & Zinc transport protein ZntB & \\
\hline 3534549..3535577 & $z n t B 2$ & Zinc transport protein $\mathrm{ZntB}$ & \\
\hline 4931416..4931847 & $z n t R$ & HTH-type transcriptional regulator ZntR & \\
\hline 220203..222914 & $\operatorname{cop} A$ & $\mathrm{Cu}+$ exporting ATPase & Copper \\
\hline $2940939 . .2941868$ & - & rhizopine-binding protein & \multirow{5}{*}{$\begin{array}{l}\text { Rhizopine degradation } \\
\text { and transport }\end{array}$} \\
\hline 2944548..2945468 & - & rhizopine-binding protein & \\
\hline $3659835 . .3660758$ & - & rhizopine-binding protein & \\
\hline 499977..501107 & iolG2 & Myo-inositol 2-dehydrogenase & \\
\hline $4613990 . .4615003$ & iolG2 & $\begin{array}{l}\text { Inositol 2-dehydrogenase/D-chiro-inositol 3- } \\
\text { dehydrogenase }\end{array}$ & \\
\hline 1188384..1189376 & dcyD & D-cysteine desulfhydrase & D-cysteine desulfhydrase \\
\hline
\end{tabular}

Source: Data from the research using the bioinformatics tools already described in the methodology.

The genes encoded in the set described in table 1 are related to several physiological processes in plants and contribute directly or indirectly to their growth and development, including mechanisms as the solubilization of minerals and siderophores that act in iron uptake.

The sequence analysis indicated that in the present Serratia marcescens genome was observed interesting genes for industries (Table 2).

Table 2. Genes involved in the antimicrobial activities or important enzymes for industry.

\begin{tabular}{|c|c|c|c|}
\hline Location & Gene & Product & Pathway \\
\hline $1490734 . .1491864$ & $\operatorname{lgrD1}$ & Linear gramicidin synthase subunit D & \multirow{4}{*}{ Gramicidin synthesis } \\
\hline 2106391..2109378 & $\operatorname{lgrD2}$ & Linear gramicidin synthase subunit D & \\
\hline $2287830 . .2289554$ & $\operatorname{lgrD3}$ & Linear gramicidin synthase subunit D & \\
\hline 3621738.. 3630248 & $\operatorname{lgrD} 4$ & Linear gramicidin synthase subunit D & \\
\hline 2321416..2324511 & tycB & Tyrocidine synthase 2 & Tyrocydine synthesis \\
\hline $3339016 . .3340038$ & ansA & L-asparaginase 1 & \multirow{2}{*}{ Asparaginase synthesis } \\
\hline 810898..811944 & $a n s B$ & L-asparaginase 2 & \\
\hline 3305129..3306745 & aprA1 & Serralysin C & \multirow{2}{*}{ Serralysin } \\
\hline 3975333..3976796 & aprA2 & Serralysin & \\
\hline 2419429..2423367 & $s w r W$ & Serrawetin W1 synthethase & Serrawettin \\
\hline 1935071..1936036 & pvcA & paerucumarin biosynthesis protein $\mathrm{PvcA}$ & Paerucumarin \\
\hline 1934111..1934974 & pvcB & pyoverdine chromophore biosynthetic protein $\mathrm{PvcB}$ & Pioverdin \\
\hline $3492125 . .3493030$ & $\mathrm{rdmC}$ & Aclacinomycin methylesterase & Aclacinomycin \\
\hline 1793656..1794648 & $\mathrm{vgb}$ & Virginiamycin B lyase & Virginiamycin \\
\hline 4541527..4542810 & $\operatorname{cod} \mathrm{A}$ & Cytosine deaminase & Cytosine \\
\hline 1932827..1934086 & rebG & 4'-demethylrebeccamycin synthase & Rebeccamycin \\
\hline 2384684..2385199 & ubiC & Chorismate pyruvate-lyase & $\begin{array}{l}\text { 4-hydroxybenzoate } \\
\text { Production }\end{array}$ \\
\hline 1087373..1088089 & - & Laccase domain protein & Laccase \\
\hline 1758635..1759366 & - & Laccase domain protein & Laccase \\
\hline
\end{tabular}




\begin{tabular}{|c|c|c|c|}
\hline $1365960 . .1366358$ & pulS & Pullulanase secretion protein & Pullulanase \\
\hline 3193302..3195146 & - & Lipase & \multirow{2}{*}{ Lipase } \\
\hline 3825243..3827231 & apeE & Lipase & \\
\hline 1304805..1305767 & $y f e X 1$ & putative deferrochelatase/peroxidase YfeX & \multirow{2}{*}{$\begin{array}{c}\text { Predicted dye- } \\
\text { decolorizing peroxidase } \\
\text { (DyP), YfeX-like } \\
\text { subgroup } \\
\end{array}$} \\
\hline 4817785.4818684 & yfeX2 & putative deferrochelatase/peroxidase YfeX & \\
\hline $160868 . .162139$ & $\operatorname{clp} X$ & ATP-dependent Clp protease ATP-binding subunit ClpX & \multirow{6}{*}{ Protease } \\
\hline $160103 . .160726$ & $\operatorname{clpP1}$ & ATP-dependent Clp protease proteolytic subunit & \\
\hline 2965578..2966171 & $\operatorname{clpP2}$ & ATP-dependent Clp protease proteolytic subunit & \\
\hline 785408..785728 & $\operatorname{clpS}$ & ATP-dependent Clp protease adapter protein ClpS & \\
\hline 2177784..2179724 & ftsH & ATP-dependent zinc metalloprotease FtsH & \\
\hline $162286 . .164688$ & lon & Lon protease & \\
\hline $503103 . .503648$ & - & antibiotic biosynthesis monooxygenase & \multirow{4}{*}{ Antibiotic biosynthesis } \\
\hline $599468 . .599815$ & - & antibiotic biosynthesis monooxygenase & \\
\hline $905395 . .905697$ & - & antibiotic biosynthesis monooxygenase & \\
\hline $2877296 . .2877583$ & - & antibiotic biosynthesis monooxygenase & \\
\hline 2719913..2721460 & $\operatorname{sacC}$ & Levanase & Levanase \\
\hline $3493198 . .3494697$ & - & Carboxypeptidase M32 & Carboxipeptidase \\
\hline $128463 . .129065$ & $\begin{array}{c}a h p C / P \\
r x 1\end{array}$ & Alkyl hydroperoxide reductase $\mathrm{C}$ & \multirow{4}{*}{$\begin{array}{l}\text { Redox-relevant proteins } \\
\text { Peroxiredoxin }\end{array}$} \\
\hline $505946 . .506422$ & $\begin{array}{c}\text { OsmC/ } \\
\text { Ohr }\end{array}$ & OsmC/Ohr family protein & \\
\hline 4681856..4682587 & $\operatorname{Prx} 5$ & Hybrid peroxiredoxin hyPrx 5 & \\
\hline $4260562 . .4261026$ & Bcp & Peroxiredoxin Bcp & \\
\hline 1630232..1631041 & oxyRl & Hydrogen peroxide-inducible genes activator & \multirow{22}{*}{ Redox-relevant proteins } \\
\hline $2417256 . .2418143$ & oxyR2 & Hydrogen peroxide-inducible genes activator & \\
\hline 4680787..4681704 & oxyR3 & Hydrogen peroxide-inducible genes activator & \\
\hline $793069 . .794040$ & $\operatorname{tr} x B$ & Thioredoxin reductase & \\
\hline 3922748..3923074 & $\operatorname{trx} A$ & thioredoxin-1 & \\
\hline 4036817..4037236 & $\operatorname{trx} C$ & thioredoxin-2 & \\
\hline $3685812 . .3687032$ & - & FMN oxidoreductase / NADH oxidase & \\
\hline $1426084 . .1428279$ & katG & Catalase-peroxidase & \\
\hline $1602395 . .1603831$ & katA & Catalase & \\
\hline 3132548..3133099 & btuE & Thioredoxin/glutathione peroxidase BtuE & \\
\hline $555140 . .555643$ & Dps & DNA protection during starvation protein & \\
\hline $3182642 . .3183220$ & $\operatorname{sod} B$ & Superoxide dismutase $[\mathrm{Fe}]$ & \\
\hline 3195388..3195909 & $\operatorname{sod} C$ & Superoxide dismutase $[\mathrm{Cu}-\mathrm{Zn}]$ & \\
\hline $3842725 . .3843345$ & $\operatorname{sod} A$ & Superoxide dismutase $[\mathrm{Mn}]$ & \\
\hline $748967 . .749230$ & grxA & Glutaredoxin 1 & \\
\hline $1065094 . .1065741$ & grxB & Glutaredoxin 2 & \\
\hline 3184654..3185001 & $\operatorname{grxD}$ & Glutaredoxin 4 & \\
\hline $4062518 . .4062748$ & $n r d H$ & Glutaredoxin-like protein $\mathrm{NrdH}$ & \\
\hline $4668552 . .4669904$ & Gor & Glutathione reductase & \\
\hline 1305840..1306949 & $y b d K$ & Putative glutamate--cysteine ligase 2 & \\
\hline $1774900 . .1776462$ & $g \operatorname{sh} A$ & Glutamate--cysteine ligase & \\
\hline $891799 . .892272$ & SoxR & Redox-sensitive transcriptional activator SoxR & \\
\hline 2314122..2317031 & - & Non Ribossomal Peptide Synthetase & NRPS \\
\hline 2141301..2143492 & - & CRISPR with 37 repeat units & \multirow{2}{*}{ Crispr } \\
\hline 2151971..2152778 & - & CRISPR with 14 repeat units & \\
\hline $2060837 . .2062021$ & thlA & Beta-ketothiolase & Butanol Biosynthesis \\
\hline 1699934..1701244 & fadI & 3-ketoacyl-CoA thiolase FadI & \multirow{5}{*}{ Fosmidomicyn } \\
\hline $4021254 . .4022417$ & fadA & 3-ketoacyl-CoA thiolase & \\
\hline $2059895 . .2060818$ & $H b d$ & 3-hydroxybutyryl-CoA dehydrogenase & \\
\hline 4858741..4859607 & paaF & 2,3-dehydroadipyl-CoA hydratase & \\
\hline $2291302 . .2293044$ & - & putative acyl-CoA dehydrogenase fadE25 & \\
\hline
\end{tabular}




\begin{tabular}{|c|c|c|c|}
\hline $2293034 . .2294734$ & - & Cyclohexane-1-carbonyl-CoA dehydrogenase & \\
\hline $3359406 . .3362078$ & adhE & Aldehyde-alcohol dehydrogenase & \\
\hline $3716600 . .3718120$ & ald1 & Long-chain-aldehyde dehydrogenase & \\
\hline $2617805 . .2618833$ & adhl & Alcohol dehydrogenase & \\
\hline $3670580 . .3671665$ & adh2 & Alcohol dehydrogenase & \\
\hline 3718188..3719204 & $\operatorname{adh} A$ & Alcohol dehydrogenase 1 & \\
\hline $213877 . .215097$ & $f s r$ & Fosmidomycin resistance protein & \\
\hline $251867 . .253057$ & bcrl & Bicyclomycin resistance protein & \multirow{2}{*}{ Bicyclomycin } \\
\hline $1540827 . .1542023$ & $b c r 2$ & Bicyclomycin resistance protein & \\
\hline
\end{tabular}

Source: Data from the research using the bioinformatics tools already described in the methodology.

The wide variety of gene repertoire allows $S$. marcescens to arouse the interest of different branches of the industry, especially food, chemical, and pharmaceuticals. In the latter, the emphasis would be on agents with antimicrobial capacity that are increasingly important in this sector.

Some sets of genes can indirectly support plant growth promotion. Table 3 displays genes that enable the degradation of xenobiotics substances in the environment.

Table 3. Selected metabolism xenobiotics genes.

\begin{tabular}{|c|c|c|c|}
\hline Position & Gene & Product & Pathway \\
\hline $244791 . .245300$ & $b p h C$ & Biphenyl-2,3-diol 1,2-dioxygenase & \multirow[t]{2}{*}{ Biphenyl degradation } \\
\hline $3359406 . .3362078$ & bphJ2 & Aldehyde-alcohol dehydrogenase & \\
\hline 1431084..1432136 & - & nitronate monooxygenase & Degradation of nitronates \\
\hline 270893..271597 & azoR1 & FMN-dependent NADH-azoreductase & \multirow{3}{*}{ Degradation of azo dyes } \\
\hline 1197216..1197815 & azoR2 & FMN-dependent NADH-azoreductase & \\
\hline 3483743..3484348 & azoR3 & FMN-dependent NADH-azoreductase & \\
\hline 1385273..1385860 & - & Nitroreductase & $\begin{array}{l}\text { Degradation of nitro } \\
\text { compounds }\end{array}$ \\
\hline 2028972..2030684 & atsA & Arylsulfatase & \multirow{2}{*}{$\begin{array}{l}\text { Degradation of aryl } \\
\text { sufates }\end{array}$} \\
\hline 2789519..2791282 & - & Arylsulfatase & \\
\hline $3296973 . .3297431$ & iorA & $\begin{array}{l}\text { isoquinoline 1-oxidoreductase alpha subunit (EC } \\
1.3 .99 .16)\end{array}$ & \multirow{2}{*}{$\begin{array}{c}\mathrm{N} \text {-heterocyclic aromatic } \\
\text { compound } \\
\text { degradation }\end{array}$} \\
\hline $3294751 . .3296973$ & iorB & isoquinoline 1-oxidoreductase beta subunit (EC 1.3.99.16) & \\
\hline $2434314 . .2434766$ & aroQ & 3-dehydroquinate dehydratase & \multirow{2}{*}{ Quinate degradation } \\
\hline $4552790 . .4553890$ & aroB & 3-dehydroquinate synthase & \\
\hline $598436 . .599455$ & - & Cytochrome P450 & \multirow{2}{*}{$\begin{array}{c}\text { Metabolism of } \\
\text { xenobiotics by } \\
\text { cytochrome P450 }\end{array}$} \\
\hline $2295600 . .2296796$ & - & Cytochrome P450 & \\
\hline $1819025 . .1820365$ & pcaK & 4-hydroxybenzoate transporter PcaK & \multirow{2}{*}{$\begin{array}{c}\text { Hydroxybenzoate } \\
\text { degradation }\end{array}$} \\
\hline 4302728..4304077 & mhbT & 3-hydroxybenzoate transporter MhbT & \\
\hline 1333277..1335343 & paaZ & Bifunctional protein $\mathrm{PaaZ}$ & \multirow{14}{*}{$\begin{array}{l}\text { Phenylacetate } \\
\text { degradation }\end{array}$} \\
\hline $1335661 . .1336599$ & paaA & 1,2-phenylacetyl-CoA epoxidase, subunit A & \\
\hline $1336621 . .1336908$ & paaB & 1,2-phenylacetyl-CoA epoxidase, subunit B & \\
\hline 1336917..1337675 & paaC & 1,2-phenylacetyl-CoA epoxidase, subunit C & \\
\hline $1337685 . .1338182$ & paaD & Putative 1,2-phenylacetyl-CoA epoxidase, subunit D & \\
\hline 1338196..1339254 & paaE & 1,2-phenylacetyl-CoA epoxidase, subunit E & \\
\hline $1339263 . .1340036$ & paaF1 & 2,3-dehydroadipyl-CoA hydratase & \\
\hline $1340040 . .1340831$ & paaG & 1,2-epoxyphenylacetyl-CoA isomerise & \\
\hline 1340834..1342363 & paaH & 3-hydroxyadipyl-CoA dehydrogenase & \\
\hline 1342360..1342800 & paaI & Acyl-coenzyme A thioesterase PaaI & \\
\hline 1342797..1344002 & paaJ & 3-oxoadipyl-CoA/3-oxo-5,6-dehydrosuberyl-CoA thiolase & \\
\hline 1344018..1345325 & paaK & Phenylacetate-coenzyme A ligase & \\
\hline 1345404..1346342 & paaX & Transcriptional repressor $\mathrm{PaaX}$ & \\
\hline 4858741..4859607 & paaF2 & 2,3-dehydroadipyl-CoA hydratase & \\
\hline
\end{tabular}




\begin{tabular}{|c|c|c|c|}
\hline $3548984 . .3550480$ & caeA & \multirow{4}{*}{ Carboxylesterase } & \multirow{4}{*}{$\begin{array}{l}\text { Organophosphates, } \\
\text { Carbamates and } \\
\text { Pyrethroids Degradation }\end{array}$} \\
\hline 3703319..3704146 & ybfK & & \\
\hline 1108361..1109335 & nhl1 & & \\
\hline 3730397...3731275 & nhl2 & & \\
\hline $499125 . .499748$ & gstB1 & \multirow{4}{*}{ Glutathione S-transferase } & \multirow{14}{*}{$\begin{array}{l}\text { Degradation of various } \\
\text { xenobiotics compounds }\end{array}$} \\
\hline $525352 . .526008$ & gstB2 & & \\
\hline $713294 . .713914$ & gstB3 & & \\
\hline 1351981..1352598 & gstB4 & & \\
\hline $903120 . .903623$ & yqjA1 & Inner membrane protein YqjA & \\
\hline $2535596 . .2536276$ & yqjA2 & Inner membrane protein YqjA & \\
\hline 2534611..2534982 & yqjC & Protein YqjC & \\
\hline $2534141 . .2534446$ & yqjD1 & putative protein YqjD & \\
\hline $4065045 . .4065353$ & yqjD2 & putative protein YqjD & \\
\hline $2531706 . .2532692$ & yqjG & Glutathionyl-hydroquinone reductase YqjG & \\
\hline $2533737 . .2534138$ & yqjE & Inner membrane protein YqjE & \\
\hline 2532763..2533158 & yqjF & Inner membrane protein $\mathrm{YqjF}$ & \\
\hline $309356 . .310000$ & yqjI & Transcriptional regulator YqjI & \\
\hline $599468 . .599815$ & yqjZ & putative protein YqjZ & \\
\hline
\end{tabular}

Source: Data from the research using the bioinformatics tools already described in the methodology.

Healthy environmental conditions are essential for plant survival and growth. For example, the set of genes in Table 3 supports the degradation of substances discarded by industries, such as synthetic textile dyes and heavy metals, helping to support the soil quality.

Growth-promoting bacteria that have the ability to survive in places with the presence of antibiotics and heavy metals have greater added value. Table 4 presents some relevant genes in this context.

Table 4. Genes responsible for the resistance to different metals and antibiotics.

\begin{tabular}{|c|c|c|c|}
\hline Position & Gene & Product & Pathway \\
\hline $3559798 . .3561174$ & $c z c D$ & Cobalt-zinc-cadmium resistance protein & Cobalt-zinc-cadmium \\
\hline 3259449..3259841 & - & transcriptional regulator & resistance \\
\hline $4722901 . .4724295$ & cpxA1 & Sensor histidine kinase CpxA & \multirow{5}{*}{$\begin{array}{l}\text { Multidrug Resistance } \\
\text { Cascade }\end{array}$} \\
\hline 4824636..4825931 & cpxA2 & Sensor histidine kinase CpxA & \\
\hline 4721578..4722057 & $\operatorname{cpxP}$ & Periplasmic protein CpxP & \\
\hline $4722206 . .4722904$ & cpxR1 & Transcriptional regulatory protein $\mathrm{CpxR}$ & \\
\hline $4823922 . .4824602$ & cpxR2 & Transcriptional regulatory protein CpxR & \\
\hline $4231962 . .4233395$ & $m g t E$ & Magnesium transporter MgtE & Magnesium transporter \\
\hline 277974..278876 & $\operatorname{cor} C$ & Magnesium and cobalt efflux protein CorC & $\begin{array}{c}\text { Magnesium and cobalt efflux } \\
\text { protein } \\
\end{array}$ \\
\hline $3972332 . .3974650$ & $z n t A$ & zinc/cadmium/mercury/lead-transporting ATPase & $\begin{array}{c}\text { Zinc/cadmium/mercury/lead } \\
\text {-transporting }\end{array}$ \\
\hline 356111..357073 & $z n t B$ & zinc transporter ZitB & \multirow{4}{*}{ Zinc transporter } \\
\hline 1039135..1040082 & $z n u A$ & zinc $\mathrm{ABC}$ transporter substrate-binding protein & \\
\hline 1040158..1040916 & $z n u C$ & zinc import ATP-binding protein ZnuC & \\
\hline 1040913..1041698 & $z n u C$ & zinc $\mathrm{ABC}$ transporter permease & \\
\hline $220203 . .222914$ & $\operatorname{cop} A$ & Copper-exporting P-type ATPase & \multirow{9}{*}{ Copper Resistance } \\
\hline $1054097 . .1054855$ & cutC & copper homeostasis protein CutC & \\
\hline 1679204..1679566) & $\operatorname{scs} A$ & copper resistance protein & \\
\hline $2927427 . .2928308$ & Crd & copper resistance protein D & \\
\hline 4495263..4495946 & cutF & copper homeostasis/adhesion lipoprotein NlpE & \\
\hline $1679204 . .1679566$ & $\operatorname{scs} A$ & copper resistance protein & \\
\hline 1677118..1679154 & $s \operatorname{cs} B$ & protein-disulfide reductase & \\
\hline $1676405 . .1677121$ & $\operatorname{scs} C$ & thioredoxin domain-containing protein & \\
\hline 1675912..1676412 & $s c s D$ & protein disulfide oxidoreductase & \\
\hline $2560078 . .2560518$ & aac6'-Ic & Aminoglycoside acetyltransferase & Aminoglycoside \\
\hline $3238359 . .3239495$ & srt-2 & Beta-lactamase & Cefotaxime, cephalosporin \\
\hline 3578532..3579713 & tet(41) & Tetracycline efflux pump & Tetracycline \\
\hline 4526805..4527437 & crp & Regulator of MdtEF multidrug efflux pump expression. & $\begin{array}{c}\text { Fluoroquinolone, macrolide, } \\
\text { penam }\end{array}$ \\
\hline
\end{tabular}




\begin{tabular}{cclc}
\hline $\mathbf{8 9 3 5 1 4 . . 8 9 6 5 3 8}$ & mexI & Inner membrane transporter of the efflux complex MexGHI-OpmD & $\begin{array}{c}\text { Fluoroquinolone, } \\
\text { tetracycline }\end{array}$ \\
\hline $\mathbf{2 9 0 7 6 2 7 . . 2 9 1 0 7 4 7}$ & oqxB & Efflux pump & $\begin{array}{c}\text { Fluoroquinolone, } \\
\text { glycylcycline, nitrofuran, } \\
\text { tetracycline }\end{array}$ \\
\hline $\mathbf{3 3 5 7 5 9 4 . . 3 3 5 7 9 9 5}$ & h-ns & Repressor of the membrane fusion protein genes & $\begin{array}{c}\text { Cephalosporin, cephamycin, } \\
\text { fluoroquinolone, macrolide, } \\
\text { penam, tetracycline }\end{array}$ \\
\hline
\end{tabular}

Source: Data from the research using the bioinformatics tools already described in the methodology.

Bacterial resistance to metals and antimicrobials present in S. marcescens can bring advantages and allow the plant to survive in environments with multiple stresses, making the bacteria attractive, even for use in bioremediation of the environment.

Complementary characteristics in PGPR bacteria, such as the ability to defend against plant parasites, which can play an important role, are described in table 5.

Table 5. Genes responsible for the nematicidal and larvicidal kill genes.

\begin{tabular}{|c|c|c|c|}
\hline Position & Gene & Product & Pathway \\
\hline 4140944..4142197 & glyA & Serine hydroxymethyl transferase & \multirow{4}{*}{ Acetaldehyde } \\
\hline $3359406 . .3362078$ & adhE & Aldehyde-alcohol dehydrogenase & \\
\hline $3670580 . .3671665$ & $a d h$ & Alcohol dehydrogenase & \\
\hline $3045834 . .3047330$ & рииC & NADP/NAD-dependent aldehyde dehydrogenase PuuC & \\
\hline $919496 . .919756$ & hcnA & Hydrogen cyanide synthase subunit HcnA & \multirow{3}{*}{ Cyanide } \\
\hline 919749..921017 & $h c n B$ & Hydrogen cyanide synthase subunit HcnB & \\
\hline 918375..919499 & hcnC & Hydrogen cyanide synthase subunit HenC & \\
\hline $1655365 . .1656882$ & purF & Amidophosphoribosyltransferase & $\begin{array}{c}\text { Member of the } \\
\text { purine/pyrimidine } \\
\text { phosphoribosyltransferas } \\
\text { e family }\end{array}$ \\
\hline $3336851 . .3338128$ & chiAl & Chitinase & \multirow{4}{*}{ Chitin degradation } \\
\hline 3889421..3891112 & chiA & Chitinase & \\
\hline 4797788..4799287 & chiB & Chitinase & \\
\hline $286807 . .288246$ & chid & Chitinase & \\
\hline $2419429 . .2423367$ & $s w r W$ & Serrawetin W1 synthethase & Serrawettin \\
\hline
\end{tabular}

Source: Data from the research using the bioinformatics tools already described in the methodology.

The presence of compounds of microbial origin with insecticidal and larvicide potential is interesting for plant growth promoter candidates because it also allows its use in biocontrol activity.

\section{Discussion}

\subsection{Genes involved in the plant growth promotion traits}

In the present Serratia marcescens genome was observed genes related to several systems involved in plant-growth promotion (table 1). Among the systems found, the Tryptophan biosynthesis pathway, a system related to several physiological processes in plants, including the biosynthesis of phytohormone indole acetic acid (IAA) (Khan et al., 2017), another system also observed in the present sample. Unfortunately, IAA biosynthesis does not have its mechanism fully elucidated. However, it is known that phytohormones such as auxins and cytokinins are involved in critical physiological processes of plants such as cell wall elongation and cell division stimulus, respectively (Xie et al., 2020). The IAA can act as an inducer compound of Induced Systemic Resistance (ISR). The ISR is an important plant defense mechanism against a broad range of pathogens, 
parasitic weeds, and even insect herbivores. PGPR producing IAA may enhance the resistance against pathogens. The ISR has already been shown to be effective in rhizobacterium S. marcescens acting as a PGPR (Ryu et al., 2013).

The present genome also contains genes that express the non-protein amino acid gamma-aminobutyric acid (GABA), a well-known neurotransmitter in the mammalian body first isolated in 1949, but with less than 20 years of study in plant organisms. Until no less than six years ago, the influence of GABA in the plant-growth was described (Ramesh et al., 2017) identify a plant 'GABA receptor' that inhibits anion passage through the aluminum-activated malate transporter family of proteins (ALMT) and propose that GABA regulation of ALMT activity could function as a signal that modulates plant growth, development, and stress response.

This study also demonstrates other growth promoters in plants that act indirectly, such as the biosynthesis and transport capacity of several polyamines, more precisely putrescine, cadaverine, and spermine, which stimulate the endogenous production of growth promoters such as IAAs and reducing growth inhibitors (A. A. Amin et al., 2011). Chitin degradation is also an indirect growth mechanism present in this Serratia strain, proving to be an important factor in several microbiological agents in controlling pathogenic fungi in several plant species (Das et al., 2010). The capacity of acetoin synthesis complements promoting indirect growth as a precursor agent of volatile bacterial compounds (Sharifi \& Ryu, 2018).

It also has a mechanism for the production of siderophores, organic compounds that act in iron uptake. Since iron is abundant in its insoluble form in the soil, siderophores act on the excretion of substances, forming soluble compounds with iron and then being absorbed (Parmar \& Chakraborty, 2016). The siderophores that the present strain produces are Bacterioferritin, enterobactin, and isochorismate.

Another system for promoting plant growth found in this genome involving the solubilization of minerals is the Phosphate solubilization system, both organic and inorganic. Phosphate is commonly found in the soil in its insoluble form (Rodríguez \& Fraga, 1999), so the performance of these mechanisms becomes essential for the absorption of the mineral. It is worth noting that the present strain also contains the gcd and pqq gene complex, which respectively code for glucose dehydrogenase and pyrroloquinoline quinone, also called the quinoprotein glucose dehydrogenase complex. A complex that acts in the release of gluconic acid in the soil, helping in the solubilization of inorganic phosphate. Highlighting that the pqq gene acts as a cofactor for the performance of glucose dehydrogenase (Abreo \& Altier, 2019). Although phosphate is rarely present in its organic form in the soil, with average measurements around $1 \mathrm{ppm}$ or even less in some cases, its role is also crucial in promoting plant growth. To be absorbed, soil bacteria act by hydrolyzing organic phosphate to inorganic phosphate and then proceed to absorption with the originally inorganic phosphate (Rodríguez \& Fraga, 1999).

Still, on mineral solubilization, the present $S$. marcescens genome has zinc solubilization genes, which in turn have a direct relationship with gross growth. Bearing in mind that zinc deficiency can cause plant growth retardation, chlorosis, reduced leaf size, greater susceptibility to heat and fungal infections, it can affect grain and pollen yield, water uptake, and, in some plant species, the yellowing of the leaves (Kamran et al., 2017). Moreover, the solubilization of zinc also ends up helping in the production of siderophores.

Other genes presented by this strain promote chemotaxis activity. This activity is important because it is known that PGPR tends to have positive chemotaxis towards higher concentrations of sugars, thus being able to bring more substrates to plants and directly influence growth (Pedraza et al., 2010).

\subsection{Genes involved in antimicrobial activities or important enzymes for industry}

The functional diversity of the $S$. marcescens genome presents genes of interest for the pharmaceutical and medical sector, likewise the food, cosmetics, and fuel industries (Table 2). 
Among some products, we can highlight the versatile enzyme L-asparaginase that includes the medical application of its antimicrobial and anti-inflammatory properties besides its use as a chemotherapeutic agent in different cancer cell lines (Abdel- Razik et al., 2019). The strategy of using novel therapeutic agents isolated from S. marcescens has been previously demonstrated in a marine strain with antioxidant and antibacterial activity against some Gram-positive and Gram-negative bacteria. (Gangadharan et al., 2020).

The food sector benefits from L-asparaginase mitigation effect due to toxic acrylamide, making it an essential component in food processing industries. This sector can demand the enzyme either considering its application in plant growth promotion. The L-Asparagine is the most abundant metabolite for the storage and transport of nitrogen in plants and impacts vegetable production. The L-asparaginase breaks down asparagine into aspartic acid and ammonia, providing nutrition directly and indirectly as a precursor of other amino acids that stimulate plant growth. Furthermore, the L-asparaginase is essential for developing seeds and grains (Damare \& Kajawadekar, 2020).

The antimicrobial property is also of particular interest in Gramicidin D, a natural antimicrobial peptide produced by the soil microorganism Bacillus brevis ATCC 8185 during its sporulation phase. This ionophoric antibiotic forms membrane channels resulting in pores' formation, leading to cell disruption and loss of solutes and ions. The Gramicidin D has been effective against antibiotic resistant microorganisms, inhibiting the genetic material synthesis and respiration and reducing ATP, leading to cell death (Pavithrra \& Rajasekaran, 2020).

The high hemolytic activity of Gramicidin D turns it more suitable where there is low hemolytic activity, as in antimalarial activity against Plasmodium falciparum (Gumila et al., 1997) and plants with some benefits already been demonstrated in oat roots (Hodges et al., 1971).

The food sector can also benefit from the presence of the peptide tyrocidine that has an unexplored antifungal effect against some agronomically relevant fungal phytopathogens (Troskie et al., 2014). The tyrocidines can be associated with gramicidin, either encoded by our S. marcescens strain in an antimicrobial compound called tyrothricin that shows activity against bacteria, fungi, and some viruses. This antibiotic demonstrated a low risk of resistance development in vitro, turning it a valuable therapeutic option to consider against the antibiotic resistance process (Stauss-Grabo et al., 2014).

Additionally, even new strategies proposed to treat antibiotic resistance, like bacterial predation, effective against some Gram-negative bacteria (Rames, 2020), find a barrier in our strain as a result of the metalloprotease serralysin that can reduce the ability of some predators to attach to the $S$. marcescens, conferring protection (Garcia et al., 2018). Serralysin, a virulence factor used medicinally as a proteolytic enzyme, is therapeutically useful in the management of pain and inflammation as a broad spectrum anti-inflammatory drug (Tiwari, 2017), digests non-living tissue, blood clots, cysts, and arterial plaque (Rouhani et al., 2020) and has an anticancer potential (Araghi et al., 2019).

Our S. marcescens sample revealed the peroxiredoxins OsmC/Ohr, Prx5, and Prx6 proteins. Peroxiredoxins are ubiquitous peroxidases that play an important role in antioxidant defense and regulating cell signaling pathways (Perkins et al., 2015). Ohr is involved in the host-pathogen interface, while OsmC participates more in the oxidative defense (Alegria et al., 2017). Moreover, Prx5 and Prx6 are being related to cancer prevention, although more studies are necessary to understand the interaction pattern with cancer cells (Forshaw et al., 2019).

For the best of our knowledge, all the four proteins associated with peroxiredoxins discussed above (OsmC/Ohr, Prx5, and Prx6) were not reported in Serratia marcescens before, revealing its potential and possible use in the host-pathogen interface and cancer management.

The use of substances produced by the Serratia genus and present in our lineage has benefits, including agronomy. Plant protecting properties of the biosurfactant serrawettin, for example, turns the S. marcescens an interesting candidate for controlling and preventing Oomycete pathogens infestation of plants (Strobel et al., 2002). Mechanisms of plant growth 
promotion include the synthesis of siderophores which can solubilize and sequester iron from the soil. The presence of the siderophore pyoverdine may provide iron to plants. This supply helps the bacteria to protect plants against the inhibitory effects of high concentrations of nickel, lead, and zinc (Burd et al., 2000).

The S. marcescens genome also carries genes encoding enzymes like Laccase, a component of prokaryotic lignindegrading systems. This ligninolytic genetic repertoire is reinforced by the gene YefX encoding dye-decolorizing peroxidase (DyP). This heme peroxidase is more efficient in degrading lignin than classical peroxidase considering the ability of DyP to degrade aromatic compounds that constitute approximately $90 \%$ of the lignin (Melo-Nascimento et al., 2020). Thus, the industry can benefit from lignin uses. Additions of alkali lignin to pet and human food can be an important fiber source, especially considering that high nutritional fiber relates to low occurrences of colon câncer (Naseem et al., 2016).

The DyP ability to degrade lignin can benefit its utilization as a carbon source. Considering that lignin is one of the most abundant organic macromolecules in the biosphere, it can constitute a renewable carbon feedstock, potentially reducing the use of petroleum-derived chemicals (Brown \& Chang, 2014).

The dye-decolourizing activity that gives name to the peroxidase may also benefit the industry operating in bioremediation, degrading synthetic dyes, and remediating phenolic environmental pollutants. Particularly important considering that synthetic dyes are employed in diverse industries such as food, textile, plastics, food, and pharmaceutical (Falade and Ekundayo, 2021). Bioremediation strategies using S. marcescens have been developed as a promising alternative for marine ecosystems impacted by petroderivatives (dos Santos et al., 2021).

The versatility is also present in Lipases, ubiquitous enzymes that hydrolyze ester bonds of triglycerides at oil-water. Its enantioselectivity in biocatalytic hydrolysis is being used in the pharmaceutical industry to produce key intermediates of the diltiazem hydrochloride used in circulatory disorders pharmacos and the anti-inflammatory ketoprofen (Long et al., 2007; Shibatani et al., 2000). Another application of the enzyme is its use to produce monoacylglycerols and diacylglycerols, which have the advantage of being biodegradable and non-toxic and are widely used as emulsifiers in food, pharmaceutical, and cosmetic industries (Zied et al., 2018). In addition, the lipases are known for their ability in the industry of oil and lipid processing, detergent production, and biotransformation (García-Silvera et al., 2018; Zied et al., 2018). Some studies have shown some applicability for $S$. marcescens lipase, such as petroleum biodegradation regenerating contaminated areas, generation of emulsifiers from cheap vegetable oils, and biodiesel production (García-Silvera et al., 2018; Peixoto et al., 2017; Zied et al., 2018).

Some microorganisms have been using the Butanol biosynthesis system for biofuel production. Butanol is a sustainable technology for alternate and renewable energy, being less corrosive and with higher energy content per unit mass than traditional fuels. The butanol potential includes its use as a solvent and a platform chemical in the cosmetic and pharmaceutical industry (Lv et al., 2021).

\subsection{Selected metabolism of xenobiotics genes}

\subsubsection{Degradation of azo dyes and hydroxybenzoate degradation}

There is a need for healthy environmental conditions, such as water purity and soil integrity and strength, to promote plant growth. The global aquatic resources undergo endless threats due to the discharge of several substances such as synthetic textile dyes and heavy metals, mainly from industries (Mahmood et al., 2017).

The azo dyes are a group of synthetic chemicals present in wastewaters dumped by textile industries. The water contaminated by this group of dyes affects the nearby waters used for agriculture and other purposes. Moreover, it is hard to remove from agricultural soils because of their complex structure (Mahmood et al., 2017). 
Following these lines, the contaminated water irrigates plants that incorporate these chemical substances bringing harmful effects both for the plant and for the consumer (Mahmood et al., 2017).

Our group found in the $S$. marcescens genome the system of degradation azo dyes (Table 3). It is of common knowledge that this system is responsible for decolorized azo dyes through NADH-ubiquinone: oxidoreductase enzyme activity. This group of dyes is associated with impaired plant metabolism, health, and growth (Mahmood et al., 2017).

The $S$. marcescens use may provide the soil and plant azo dyes decontamination, which can be associated with plant growth and metabolism improvement. Besides, healthy soil is essential to promote a whole agriculture quality (Ahmed et al., 2016).

Plants naturally contain relevant levels of phenolic compounds that are essential to many metabolism events, such as growth, reproduction, and protection against pathogens (Wang et al., 2015). In these lines, the $S$. marcescens genome showed a hydroxybenzoate degradation gene set, which must be considered.

\subsubsection{Degradation of nitro compounds, degradation of arylsufatase and $\mathbf{N}$-heterocyclic aromatic compound degradation}

Other harmful substances are released in the environment, such as 2,4,6-Trinitrotoluene (TNT), released from demilitarization facilities. This compound is highly toxic and hazardous to all organisms, plants, and hu mans included. The nitroreductase protein family is involved in reducing nitrogen-containing compounds, such as TNT (You et al., 2015). Some bacteria strains are already used in plants as a detoxifying tool for this kind of environment. Here we report the presence of the nitroreductase protein family in the $S$. marcescens genome, which can also be used for the plant environment decontamination and improvement.

It is common knowledge that arylsulfatase is important to $\mathrm{SO}_{4}{ }^{2-}$ plant uptake throughout the mineralization of organic sulfur (S) to $\mathrm{SO}_{4}{ }^{2-}$ and it is from bacteria strains (Knauff, Schulz, \& Scherer, 2003). Sulfur plays a fundamental role in plant metabolic processes and protein production, which prompts the plant growth or development and filling of grains, for example (Hawkesford, 2007). The $\mathrm{S}$ absence is harmful to the plant, and the arylsulfatase is essential to provide this nutrient for the plant (Knauff et al., 2003).

Besides, heterocyclic compounds are a group of chemicals also found in the environment. Some of these compounds can accumulate in the soil, leading to toxicity to plants and humans (Seo, Keum, \& Li, 2009). The S. marcescens genome sequencing showed the arylsulfatase system and N-heterocyclic aromatic compound degradation system presence, which indicates one more important role of this bacterium in promoting plant growth and health.

\subsubsection{Quinate degradation}

Quinate is an important compound produced and used by plants. It is a precursor for chlorogenic acids (CGAs) biosynthesis. The CGAs act in leaves and fruits as a protective agent against pathogens and fungus and play an antioxidant role, protecting the plant against UV radiation damage (Gritsunov et al., 2018).

The quinate degradation system is essential to provide the active compound from the quinate pathway and could be used to help in plant protection promotion, which could allow their growth and metabolism improvement. 


\subsubsection{Metabolism of xenobiotics by cytochrome P450 and hydroxybenzoate degradation and degradation of various xenobiotics compounds}

The cytochrome P450 (CYP) is an enzymatic protein superfamily found in several organisms, such as mammals, fungi, plants, and bacteria. It is composed of enzymes essential in many plant metabolic pathways, plant growth, development, and defense (Xu et al.,, 2015). The CYP family is also important in the detoxication of herbicides in plants (Stiborová et al., 2000).

Although the cytochrome P450 is already found in plants, exogenous origin use could be an essential tool for improving plant metabolism. Our group described, throughout the genome $S$. marcescens sequencing, the presence of this xenobiotics metabolism system. When applied in plant improvement, this strain could be used to deliver this system to the plant, and it can be applied in several contexts. For example, the use of contaminated soil for agriculture or plant cultures that demand high herbicide doses require a robust detox mechanism, which could not be sufficient (Gong et al., 2005). In these scenarios, the use of exogenous systems of xenobiotics metabolism could allow this kind of culture and even improve plant growth and health.

Besides, many systems of degradation of xenobiotics compounds were found in $S$. marcescens sequencing. These pathways are essential in restraining oxidative damage, xenobiotics detox, and many stress responses that are important in plant metabolism and growth (Gong et al., 2005). These systems are composed of several proteins, such as glutathione Stransferases (GSTs). Gong and colleagues (2005) showed that in vivo, the GSTs expression was related to changes in plant growth and shoot regeneration in vitro. These data demonstrate de GSTs influence in plant growth and metabolism, which could be applied in biotechnological approaches through S. marcescens usage.

\subsubsection{Phenylacetate degradation}

The Phenylacetic acid (PAA), also known as phenylacetate, belongs to a group called auxins, a class of hormones essential in plant metabolism (Cook, 2019).

PAA was described as a growth-promoting hormone, and later studies, comparing PAA with the auxin IAA, demonstrated PAA's higher activity in stimulating lateral root. The few studies with this auxin suggest PAA's predominant role in root growth and plant regular growth maintenance (Cook, 2019). Possibly PAA is important in other plant mechanisms, but more studies must deep investigate its whole function.

The S.marcescens sequencing showed a phenylacetate degradation system which must be considered in its biotechnology use, once this strain characteristic could promote the antagonist effect proposed.

\subsection{Genes responsible for the resistance to different metals and antibiotics}

The success of bioremediation is related to the bacteria's ability to survive in a contaminated environment, among others, by antimicrobials and heavy metals (Table 4). It is the case of our strain. For example, the Cpx Regulatory System Upregulates the Multidrug Resistance Cascade. The Cpx Stress Response has a global effect in a diversity of signal transduction pathways, including the bacterial resistance to antimicrobials (Guest \& Raivio, 2016).

The excessive presence of heavy metals in the environment leads to several problems in soil, such as impaired fertility, decreased microbial activity, and yield losses. This problem affects plant growth and metabolism and carries toxicity to human health (Kacálková et al., 2009). The S.marcescens sequencing showed some relevant genes in heavy metals transporting and zinc/cadmium/mercury/lead-transporting ATPase. These systems are important to transport these heavy metals and could be used in a biotechnology approach to a detox tool for plants that grow in the contaminated soil.

Besides these systems, the copper resistance system was also found in $S$. marcescens sequencing. The excessive copper accumulation in plants leads to several deleterious effects, such as reduced seed germination, impaired plant growth, low yield, 
and formation of ROS (reactive oxygen species) (H. Amin et al., 2021). Therefore, the presence of an exogenous system could control copper plant concentration and promote plant growth and health.

Metal resistance has been described in a co-selection mechanism with antibiotic resistance, particularly relevant under environmental conditions of metal stress. The selective pressure to survive under stress conditions might contribute to plant growth-promoting bacteria evolve mechanisms to tolerate the uptake of heavy metal and/or the antibiotic presence in soil, for example (Yang et al., 2021).

PGPR bacteria presenting antibiotic and metal resistance genes in parallel have been identified in environments with multiple stress in which the survivor bacterium is probably benefited by acquiring resistance to both causes of stress (Wani \& Irene, 2013).

This co-selection may occur as a co-resistance when genes encoding resistance to heavy metals and antimicrobial agents are physically linked to each other (Bazzi et al., 2020). This association has already been described in $S$. marcescens with tetracycline resistance and also to chloramphenicol and kanamycin in this case, which is genetically linked to $\mathrm{As}, \mathrm{Cu}, \mathrm{Hg}$, and Ag resistance genes (Gilmour et al., 2004).

Other ways occur with the Cross-resistance when the same resistance mechanism confers resistance to both heavy metals and antimicrobial agents. Frequently related to multi-drug efflux pumps and the least common mechanism of coselection, the Co-regulatory resistance occurs when resistant genes to antimicrobial agents and heavy metals are controlled by a mutual regulatory protein (Bazzi et al., 2020). These mechanisms of co-selection support the direct correlation between antibiotic resistance genes with the concentration of antibiotics and metals found in the manure of Chinese swine farms (Yang et al., 2021).

\subsection{Genes responsible for the nematicidal and larvicidal kill genes}

Compounds of microbial origin have been showing a positive protection effect combating plant-parasites (table 5). For example, in the root-knot nematode that impacts the global agricultural production, acetaldehyde demonstrated nematicidal activity by direct contact killing besides the fumigation inhibiting egg hatching (Huang et al., 2020).

The nematicidal effect of other substances as serrawettin and chitinase potentially produced by our strain has already been found in Serratia sp. genetically related to S. marcescens. The presence of these compounds, according to the author, is important to turn this strain into an attractive candidate as a sustainable alternative for biocontrol in crops of agricultural interest (Méndez-Santiago et al., 2021).

The broad niche and functional diversity of the S. marcescens are probably influenced by the highly dynamic genome (Cristina et al., 2019), turning relevant a periodical investigation of the genetic background from various sources, concerning its possible pathogenicity. This research collaborates in this context with the genetic repertoire of a Serratia marcescens isolated from food. In addition, the potential application of this strain as a plant growth promoter was evaluated from a genotypic perspective.

\section{Conclusion}

The S. marcescens systems described here are relevant in the plant biotechnology approach representing an alternative in promoting plant growth and health. All these systems could improve different mechanisms, both in the plants and their environment. In addition, understanding the genetic background governing this strain may bring new insights into the ecology of Serratia marcescens. 
To the best of our knowledge, this is the first genome sequence report on $S$. marcescens isolated from cheese, with potential application as promoting plant growth and providing a baseline for future genomic studies. Future investigations of others Serratia marcescens isolated from food will allow comparative analysis that may help to establish a model of the genetic background of the association between S. marcescens with plants and the food production process.

\section{References}

Abdel- Razik, N. E., EL-Baghdady, K. Z., EL-Shatoury, E. H., \& G Mohamed, N. (2019). Isolation, optimization, and antitumor activity of 1-asparaginase extracted from Pectobacterium carotovorum and Serratia marcescens on human breast adenocarcinoma and human hepatocellular carcinoma cancer cell lines. Asian Journal of Pharmaceutical and Clinical Research, 332-337. https://doi.org/10.22159/ajpcr.2019.v12i2.29646

Abreo, E., \& Altier, N. (2019). Pangenome of Serratia marcescens strains from nosocomial and environmental origins reveals different populations and the links between them. Scientific Reports, 9(1), 46. https://doi.org/10.1038/s41598-018-37118-0

Adeolu, M., Alnajar, S., Naushad, S., \& S. Gupta, R. (2016). Genome-based phylogeny and taxonomy of the 'Enterobacteriales': proposal for Enterobacterales ord. nov. divided into the families Enterobacteriaceae, Erwiniaceae fam. nov., Pectobacteriaceae fam. nov., Yersiniaceae fam. nov., Hafniaceae fam. nov., Morgane. International Journal of Systematic and Evolutionary Microbiology, 66(12), 5575-5599. https://doi.org/10.1099/ijsem.0.001485

Ahmed, F., Arshad, M., Ditta, A., Hussain, A., Naveed, M., Hasnain, M., \& Nazir, Q. (2016). Combining Textile Effluent Wastewater with Organic Fertilizer for Improved Growth and Productivity of Wheat and Soil Health. Journal of Environmental and Agricultural Sciences, 8, 14-20.

Alegria, T. G. P., Meireles, D. A., Cussiol, J. R. R., Hugo, M., Trujillo, M., de Oliveira, M. A., \& Netto, L. E. S. (2017). Ohr plays a central role in bacterial responses against fatty acid hydroperoxides and peroxynitrite. Proceedings of the National Academy of Sciences, 114(2), E132-E141. https://doi.org/10.1073/pnas.1619659114

Amarsy, R., Pean de Ponfilly, G. ., Benmansour, H. ., Jacquier, H., Cambau, E. \& Mégarbane, B. (2020). Serratia marcescens outbreak in the intensive care unit during the COVID-19 pandemic: A paradoxical risk? Médecine et Maladies Infectieuses, 50(8), 750-751. https://doi.org/10.1016/j.medmal.2020.05.004

Amin, A. A., Gharib, F. A. E., El-Awadi, M., \& Rashad, E.-S. M. (2011). Physiological response of onion plants to foliar application of putrescine and glutamine. Scientia Horticulturae, 129(3), 353-360. https://doi.org/10.1016/j.scienta.2011.03.052

Amin, H., Arain, B. A., Jahangir, T. M., Abbasi, A. R., Mangi, J., Abbasi, M. S., \& Amin, F. (2021). Copper (Cu) tolerance and accumulation potential in four native plant species: a comparative study for effective phytoextraction technique. Geology, Ecology, and Landscapes, 5(1), 53-64. https://doi.org/10.1080/24749508.2019.1700671

Araghi, A., Hashemi, S., Sepahi, A. A., Faramarzi, M. A., \& Amin, M. (2019). Purification and study of anti-cancer effects of Serratia marcescens serralysin. Iranian Journal of Microbiology, 11(4), 320-327. Retrieved from http://www.ncbi.nlm.nih.gov/pubmed/31719964

Bazzi, W., Abou Fayad, A. G., Nasser, A., Haraoui, L.-P., Dewachi, O., Abou-Sitta, G., \& Matar, G. M. (2020). Heavy Metal Toxicity in Armed Conflicts Potentiates AMR in A. baumannii by Selecting for Antibiotic and Heavy Metal Co-resistance Mechanisms. Frontiers in Microbiology, 11, 68. https://doi.org/10.3389/fmicb.2020.00068

Brettin, T., Davis, J. J., Disz, T., Edwards, R. A., Gerdes, S., Olsen, G. J., \& Xia, F. (2015). RASTtk: A modular and extensible implementation of the RAST algorithm for building custom annotation pipelines and annotating batches of genomes. Scientific Reports, 5(1), 8365. https://doi.org/10.1038/srep08365

Brown, M. E., \& Chang, M. C. Y. (2014). Exploring bacterial lignin degradation. Current Opinion in Chemical Biology, 19(1), 1-7. https://doi.org/10.1016/j.cbpa.2013.11.015

Burd, G. I., Dixon, D. G., \& Glick, B. R. (2000). Plant growth-promoting bacteria that decrease heavy metal toxicity in plants. Canadian Journal of Microbiology, 46(3), 237-245. https://doi.org/10.1139/w99-143

Cook, S. D. (2019). An Historical Review of Phenylacetic Acid. Plant and Cell Physiology, 60(2), 243-254. https://doi.org/10.1093/pcp/pcz004

Cristina, M., Sartini, M., \& Spagnolo, A. (2019). Serratia marcescens Infections in Neonatal Intensive Care Units (NICUs). International Journal of Environmental Research and Public Health, 16(4), 610. https://doi.org/10.3390/ijerph16040610

Damare, V. S., \& Kajawadekar, K. G. (2020). A preliminary study on L-asparaginase from mangrove detritus-derived fungi and its application in plant growth promotion - MycoAsia. Retrieved from http://mycoasia.org/a-preliminary-study-on-1-asparaginase-from-mangrove-detritus-derived-fungi-and-itsapplication-in-plant-growth-promotion/

Das, S. N., Dutta, S., Kondreddy, A., Chilukoti, N., Pullabhotla, S. V. S. R. N., Vadlamudi, S., \& Podile, A. R. (2010). Plant Growth-Promoting Chitinolytic Paenibacillus elgii Responds Positively to Tobacco Root Exudates. Journal of Plant Growth Regulation, 29(4), 409-418. https://doi.org/10.1007/s00344-0109152-1

Davis, J. J., Wattam, A. R., Aziz, R. K., Brettin, T., Butler, R., Butler, R. M., \& Stevens, R. (2020). The PATRIC Bioinformatics Resource Center: expanding data and analysis capabilities. Nucleic Acids Research, 48(D1), D606-D612. https://doi.org/10.1093/nar/gkz943

dos Santos, R. A., Rodríguez, D. M., Ferreira, I. N. da S., de Almeida, S. M., Takaki, G. M. de C., \& de Lima, M. A. B. (2021). Novel production of biodispersant by Serratia marcescens UCP 1549 in solid-state fermentation and application for oil spill bioremediation. Environmental Technology, 1-12. https://doi.org/10.1080/09593330.2021.1910733 
Falade, A. O., \& Ekundayo, T. C. (2021). Emerging biotechnological potentials of DyP-type peroxidases in remediation of lignin wastes and phenolic pollutants: a global assessment (2007-2019). Letters in Applied Microbiology, 72(1), 13-23. https://doi.org/10.1111/lam.13392

Forshaw, T. E., Holmila, R., Nelson, K. J., Lewis, J. E., Kemp, M. L., Tsang, A. W., \& Furdui, C. M. (2019). Peroxiredoxins in Cancer and Response to Radiation Therapies. Antioxidants, 8(1), 11. https://doi.org/10.3390/antiox8010011

Friman, M. J., Eklund, M. H., Pitkälä, A. H., Rajala-Schultz, P. J., \& Rantala, M. H. J. (2019). Description of two Serratia marcescens associated mastitis outbreaks in Finnish dairy farms and a review of literature. Acta Veterinaria Scandinavica, 61(1), 54. https://doi.org/10.1186/s13028-019-0488-7

Gangadharan, A., Jolly, J., \& John, N. (2020). Bioprospecting of novel therapeutic agents from marine bacterium; Serratia marcescens. Materials Today: Proceedings, 25, 298-301. https://doi.org/10.1016/j.matpr.2020.01.465

García-Silvera, E. E., Martínez-Morales, F., Bertrand, B., Morales-Guzmán, D., Rosas-Galván, N. S., León-Rodríguez, R., \& Trejo-Hernández, M. R. (2018). Production and application of a thermostable lipase from Serratia marcescens in detergent formulation and biodiesel production. Biotechnology and Applied Biochemistry, 65(2), 156-172. https://doi.org/10.1002/bab.1565

Garcia, C. J., Pericleous, A., Elsayed, M., Tran, M., Gupta, S., Callaghan, J. D., \& Kadouri, D. E. (2018). Serralysin family metalloproteases protects Serratia marcescens from predation by the predatory bacteria Micavibrio aeruginosavorus. Scientific Reports, 8(1), 14025. https://doi.org/10.1038/s41598-018-32330-4

Gilmour, M. W., Thomson, N. R., Sanders, M., Parkhill, J., \& Taylor, D. E. (2004). The complete nucleotide sequence of the resistance plasmid R478: defining the backbone components of incompatibility group $\mathrm{H}$ conjugative plasmids through comparative genomics. Plasmid, 52(3), 182-202. https://doi.org/10.1016/j.plasmid.2004.06.006

Gong, H., Jiao, Y., Hu, W., \& Pua, E.-C. (2005). Expression of glutathione-S-transferase and its role in plant growth and development in vivo and shoot morphogenesis in vitro. Plant Molecular Biology, 57(1), 53-66. https://doi.org/10.1007/s11103-004-4516-1

Gritsunov, A., Peek, J., Diaz Caballero, J., Guttman, D., \& Christendat, D. (2018). Structural and biochemical approaches uncover multiple evolutionary trajectories of plant quinate dehydrogenases. The Plant Journal, 95(5), 812-822. https://doi.org/10.1111/tpj.13989

Guest, R. L., \& Raivio, T. L. (2016). Role of the Gram-Negative Envelope Stress Response in the Presence of Antimicrobial Agents. Trends in Microbiology, 24(5), 377-390. https://doi.org/10.1016/j.tim.2016.03.001

Gumila, C., Ancelin, M. L., Delort, A. M., Jeminet, G., \& Vial, H. J. (1997). Characterization of the potent in vitro and in vivo antimalarial activities of ionophore compounds. Antimicrobial Agents and Chemotherapy, 41(3), 523-529. https://doi.org/10.1128/AAC.41.3.523

Hawkesford, M. J. (2007). Sulfur and plant ecology: a central role of sulfate transporters in responses to sulfur availability. https://doi.org/10.1007/978-14020-5887-5_1

Hodges, T. K., Darding, R. L., \& Weidner, T. (1971). Gramicidin-D-stimulated influx of monovalent cations into plant roots. Planta, 97(3), 245-256. https://doi.org/10.1007/BF00389205

Huang, D., Yu, C., Shao, Z., Cai, M., Li, G., Zheng, L., \& Zhang, J. (2020). Identification and Characterization of Nematicidal Volatile Organic Compounds from Deep-Sea Virgibacillus dokdonensis MCCC 1A00493. Molecules, 25(3), 744. https://doi.org/10.3390/molecules25030744

Ishii, K., Adachi, T., Imamura, K., Takano, S., Usui, K., Suzuki, K., \& Sekimizu, K. (2012). Serratia marcescens Induces Apoptotic Cell Death in Host Immune Cells via a Lipopolysaccharide- and Flagella-dependent Mechanism*. Journal of Biological Chemistry, 287(43), 36582-36592. https://doi.org/10.1074/jbc.M112.399667

Kacálková, L., Tlustoš, P., \& Száková, J. (2009). Phytoextraction of cadmium, copper, zinc and mercury by selected plants. Plant, Soil and Environment, 55(No. 7), 295-304. https://doi.org/10.17221/100/2009-PSE

Kamran, S., Shahid, I., Baig, D. N., Rizwan, M., Malik, K. A., \& Mehnaz, S. (2017). Contribution of Zinc Solubilizing Bacteria in Growth Promotion and Zinc Content of Wheat. Frontiers in Microbiology, 8(DEC), 2593. https://doi.org/10.3389/fmicb.2017.02593

Khan, A. R., Park, G.-S., Asaf, S., Hong, S.-J., Jung, B. K., \& Shin, J.-H. (2017). Complete genome analysis of Serratia marcescens RSC-14: A plant growthpromoting bacterium that alleviates cadmium stress in host plants. PLOS ONE, 12(2), e0171534. https://doi.org/10.1371/journal.pone.0171534

Knauff, U., Schulz, M., \& Scherer, H. W. (2003). Arylsufatase activity in the rhizosphere and roots of different crop species. European Journal of Agronomy, 19(2), 215-223. https://doi.org/10.1016/S1161-0301(02)00035-7

Long, Z.-D., Xu, J.-H., Zhao, L.-L., Pan, J., Yang, S., \& Hua, L. (2007). Overexpression of Serratia marcescens lipase in Escherichia coli for efficient bioresolution of racemic ketoprofen. Journal of Molecular Catalysis B: Enzymatic, 47(3-4), 105-110. https://doi.org/10.1016/j.molcatb.2007.04.004

Lv, Y., Jiang, Y., Peng, W., Fang, Y., Dong, W., Zhou, J., \& Jiang, M. (2021). Genetic manipulation of non-solvent-producing microbial species for effective butanol production. Biofuels, Bioproducts and Biorefining, 15(1), 119-130. https://doi.org/10.1002/bbb.2152

Mahmood, F., Shahid, M., Hussain, S., Shahzad, T., Tahir, M., Ijaz, M., \& Babar, S. A. K. (2017). Potential plant growth-promoting strain Bacillus sp. SR-21/1 decolorized azo dyes through NADH-ubiquinone:oxidoreductase activity. Bioresource Technology, 235, 176-184. https://doi.org/10.1016/j.biortech.2017.03.098

Melo-Nascimento, A. O. dos S., Sant`Anna, B. M. M., Gonçalves, C. C., Santos, G., Noronha, E., Parachin, N., \& Bruce, T. (2020). Complete genome reveals genetic repertoire and potential metabolic strategies involved in lignin degradation by environmental ligninolytic Klebsiella variicola P1CD1. PLOS ONE, 15(12), e0243739. https://doi.org/10.1371/journal.pone.0243739

Méndez-Santiago, E. W., Gómez-Rodríguez, O., Sánchez-Cruz, R., Folch-Mallol, J. L., Hernández-Velázquez, V. M., Villar-Luna, E., \& Wong-Villarreal, A. (2021). Serratia sp., an endophyte of Mimosa pudica nodules with nematicidal, antifungal activity and growth-promoting characteristics. Archives of 
Microbiology, 203(2), 549-559. https://doi.org/10.1007/s00203-020-02051-2

Naseem, A., Tabasum, S., Zia, K. M., Zuber, M., Ali, M., \& Noreen, A. (2016). Lignin-derivatives based polymers, blends and composites: A review. International Journal of Biological Macromolecules, 93, 296-313. https://doi.org/10.1016/j.ijbiomac.2016.08.030

Parmar, H. Y., \& Chakraborty, H. (2016). Effect of siderophore on plant growth promotion. International Journal of Applied and Pure Science and Agriculture, 2(3). Retrieved from https://ijapsa.com/papers/volume-2/issue-3/effect-of-siderophore-on-plan-growth-promotion/

Pavithrra, G., \& Rajasekaran, R. (2020). Gramicidin Peptide to Combat Antibiotic Resistance: A Review. International Journal of Peptide Research and Therapeutics, 26(1), 191-199. https://doi.org/10.1007/s10989-019-09828-0

Pedraza, R. O., Motok, J., Salazar, S. M., Ragout, A. L., Mentel, M. I., Tortora, M. L., \& Díaz-Ricci, J. C. (2010). Growth-promotion of strawberry plants inoculated with Azospirillum brasilense. World Journal of Microbiology and Biotechnology, 26(2), 265-272. https://doi.org/10.1007/s11274-009-0169-1

Peixoto, F. B. S., Peixoto, J. C. D. C., Assunção, E. N. de, Peixoto, E. M., Pereira, J. O., \& Astolfi-Filho, S. (2017). Petroleum biodegrading and co-resistance to antibiotics by Serratia marcescens strain isolated in Coari, Amazonas. Acta Scientiarum. Biological Sciences, 39(4), 489. https://doi.org/10.4025/actascibiolsci.v39i4.36223

Perkins, A., Nelson, K. J., Parsonage, D., Poole, L. B., \& Karplus, P. A. (2015). Peroxiredoxins: guardians against oxidative stress and modulators of peroxide signaling. Trends in Biochemical Sciences, 40(8), 435-445. https://doi.org/10.1016/j.tibs.2015.05.001

Rames, A. (2020). Predatory Bacteria: A Possible Key for the Lock of Antibiotic Resistance. In Transactions on Science and Technology (Vol. 7). Retrieved from http://tost.unise.org/

Ramesh, S. A., Tyerman, S. D., Gilliham, M., \& Xu, B. (2017). $\gamma$-Aminobutyric acid (GABA) signalling in plants. Cellular and Molecular Life Sciences, 74(9), 1577-1603. https://doi.org/10.1007/s00018-016-2415-7

Rodríguez, H., \& Fraga, R. (1999). Phosphate solubilizing bacteria and their role in plant growth promotion. Biotechnology Advances, 17(4-5), 319-339. https://doi.org/10.1016/S0734-9750(99)00014-2

Rouhani, M., Valizadeh, V., Molasalehi, S., \& No-Rouzian, D. (2020). Production and Expression Optimization of Heterologous Serra-tiopeptidase. Iranian Journal of Public Health, 931-939. Retrieved from http://ijph.tums.ac.ir/index.php/ijph/article/view/20663

Ryu, C.-M., Choi, H. K., Lee, C.-H., Murphy, J. F., Lee, J.-K., \& Kloepper, J. W. (2013). Modulation of Quorum Sensing in Acyl-homoserine LactoneProducing or -Degrading Tobacco Plants Leads to Alteration of Induced Systemic Resistance Elicited by the Rhizobacterium Serratia marcescens 90-166. The Plant Pathology Journal, 29(2), 182-192. https://doi.org/10.5423/PPJ.SI.11.2012.0173

Seemann, T. (2014). Prokka: rapid prokaryotic genome annotation. Bioinformatics, 30(14), 2068-2069. https://doi.org/10.1093/bioinformatics/btu153

Seo, J.-S., Keum, Y.-S., \& Li, Q. (2009). Bacterial Degradation of Aromatic Compounds. International Journal of Environmental Research and Public Health, 6(1), 278-309. https://doi.org/10.3390/ijerph6010278

Sharifi, R., \& Ryu, C.-M. (2018). Revisiting bacterial volatile-mediated plant growth promotion: lessons from the past and objectives for the future. Annals of Botany, 122(3), 349-358. https://doi.org/10.1093/aob/mcy108

Shibatani, T., Omori, K., Akatsuka, H., Kawai, E., \& Matsumae, H. (2000). Enzymatic resolution of diltiazem intermediate by Serratia marcescens lipase: molecular mechanism of lipase secretion and its industrial application. Journal of Molecular Catalysis B: Enzymatic, 10(1-3), 141-149. https://doi.org/10.1016/S1381-1177(00)00122-3

Silva, C. R., Okuno, N. T., Macedo, V. H. L. de M., Freire, I. D. R., Miller, R. M., \& Marin, V. A. (2020). Resistome in gram-negative bacteria from soft cheese in Brazil. Revista de Ciências Médicas e Biológicas, 19(3), 430. https://doi.org/10.9771/cmbio.v19i3.35460

Stauss-Grabo, M., Atiye, S., Le, T., \& Kretschmar, M. (2014). Decade-long use of the antimicrobial peptide combination tyrothricin does not pose a major risk of acquired resistance with gram-positive bacteria and candida spp. Pharmazie, 69(11), 838-841. https://doi.org/10.1691/ph.2014.4686

Stiborová, M., Schmeiser, H. ., \& Frei, E. (2000). Oxidation of xenobiotics by plant microsomes, a reconstituted cytochrome P450 system and peroxidase: a comparative study. Phytochemistry, 54(4), 353-362. https://doi.org/10.1016/S0031-9422(00)00123-0

Strobel, G. A., Morrison, S. L., \& Cassella, M. (2002). Protecting plants from oomycete pathogens by treatment with compositions containing serratamolide and oocydin a from Serratia marcescens. https://doi.org/C07D493/08

Tanizawa, Y., Fujisawa, T., Kaminuma, E., Nakamura, Y., \& Arita, M. (2016). DFAST and DAGA: web-based integrated genome annotation tools and resources. Bioscience of Microbiota, Food and Health, 35(4), 173-184. https://doi.org/10.12938/bmfh.16-003

Tanizawa, Y., Fujisawa, T., \& Nakamura, Y. (2018). DFAST: a flexible prokaryotic genome annotation pipeline for faster genome publication. Bioinformatics, 34(6), 1037-1039. https://doi.org/10.1093/bioinformatics/btx713

Tiwari, M. (2017). The role of serratiopeptidase in the resolution of inflammation. Asian Journal of Pharmaceutical Sciences, 12(3), 209-215. https://doi.org/10.1016/j.ajps.2017.01.003

Troskie, A. M., de Beer, A., Vosloo, J. A., Jacobs, K., \& Rautenbach, M. (2014). Inhibition of agronomically relevant fungal phytopathogens by tyrocidines, cyclic antimicrobial peptides isolated from Bacillus aneurinolyticus. Microbiology, 160(9), 2089-2101. https://doi.org/10.1099/mic.0.078840-0

Wang, J.-Y., Zhou, L., Chen, B., Sun, S., Zhang, W., Li, M., ... He, Y.-W. (2015). A functional 4-hydroxybenzoate degradation pathway in the phytopathogen Xanthomonas campestris is required for full pathogenicity. Scientific Reports, 5(1), 18456. https://doi.org/10.1038/srep18456 
Research, Society and Development, v. 11, n. 1, e29611124799, 2022

(CC BY 4.0) | ISSN 2525-3409 | DOI: http://dx.doi.org/10.33448/rsd-v11i1.24799

Wani, P. A., \& Irene, O. I. (2013). Screening of Microbes for Their Metal, Antibiotic Resistance and Plant Growth Promoting Activity. Current Research in Bacteriology, 7(1), 22-31. https://doi.org/10.3923/crb.2014.22.31

Xie, Q., Essemine, J., Pang, X., Chen, H., \& Cai, W. (2020). Exogenous application of abscisic acid to shoots promotes primary root cell division and elongation. Plant Science, 292, 110385. https://doi.org/10.1016/j.plantsci.2019.110385

Xu, J., Wang, X., \& Guo, W. (2015). The cytochrome P450 superfamily: Key players in plant development and defense. Journal of Integrative Agriculture, 14(9), 1673-1686. https://doi.org/10.1016/S2095-3119(14)60980-1

Yang, F., Zhang, F., Li, H., Wu, H., Zhao, H., Cheng, X., \& Zhu, J. (2021). Contribution of environmental factors on the distribution of antibiotic resistance genes in agricultural soil. European Journal of Soil Biology, 102, 103269. https://doi.org/10.1016/j.ejsobi.2020.103269

You, S.-H., Zhu, B., Han, H.-J., Wang, B., Peng, R.-H., \& Yao, Q.-H. (2015). Phytoremediation of 2,4,6-trinitrotoluene by Arabidopsis plants expressing a NAD(P)H-flavin nitroreductase from Enterobacter cloacae. Plant Biotechnology Reports, 9(6), 417-430. https://doi.org/10.1007/s11816-015-0379-y

Zied, Z., Edahech, A., Rigano, F., Micalizzi, G., Mondello, L., Kharrat, N., \& Cacciola, F. (2018). Monoacylglycerol and diacylglycerol production by hydrolysis of refined vegetable oil by-products using an immobilized lipase from Serratia sp. W3. Journal of Separation Science, 41(23), 4323-4330. https://doi.org/10.1002/jssc.201800432 